\title{
Relation between coronary artery remodelling (compensatory dilatation) and stenosis in human native coronary arteries
}

\author{
A M Varnava, M J Davies
}

\begin{abstract}
Objectives-To investigate the contribution of plaque size and vessel remodelling to coronary artery stenosis and to assess the role of vessel shrinkage (negative remodelling) across a wide range of lesions.

Design-Postmortem study of coronary remodelling in perfusion fixed hearts.

Subjects-24 men and 24 women who died suddenly with coronary artery disease.

Main outcome measures-Percentage stenosis, percentage plaque burden, percentage remodelling, and arc of normal vessel were measured and related to age, sex, smoking status, and history of hypertension.

Results-There was a positive relation between percentage stenosis and percentage plaque burden $(r=0.6, \mathrm{p}<0.0001)$ and an inverse relation between percentage stenosis and percentage remodelling $(r=-0.4, \mathrm{p}<0.0001)$. Multilinear regression modelling showed that luminal stenosis $=1.0$ (plaque burden) -0.4 (vessel remodelling). Remodelling was greater in lesions that would not have been significant at angiography ( $\leqslant 25 \%$ stenosis) than in the remaining lesions $(25.9(26) \%$ v $10.0(21.1) \%, \mathrm{p}<0.0001$, respectively) and was reduced in segments with circumferential plaques $(12.7(24.5) \% v 20.7(24.3) \%$ in eccentric plaques, $\mathrm{p}=0.001)$. Remodelling did not correlate with age, sex, or smoking. Negative remodelling was present in 62 lesions with a stenosis $>25 \%$ versus 10 lesions with $\leqslant 25 \%$ stenosis $(p<0.0001)$. Lesions with negative remodelling had greater plaque burden and luminal stenosis and a reduced arc of normal segment.

Conclusion-Outward arterial remodelling negates the stenosing effect of increasing plaque size. Significant coronary stenoses arise from a failure of this outward remodelling in the face of a large plaque burden. Coronary arterial remodelling is unrelated to sex or smoking and is plaque specific.
\end{abstract}

(Heart 2001;86:207-211)

Keywords: coronary artery disease; vessel remodelling; pathology

As an atherosclerotic plaque develops, the coronary artery increases its cross sectional area in order to preserve the normal lumen size. This process of vessel wall remodelling was described by Glagov and colleagues ${ }^{1}$ in a pathological study of human coronary arteries and has since been confirmed in vivo by intravascular ultrasound. ${ }^{2-4}$ As a result of this work the model of a simple linear relation between plaque growth and coronary artery stenosis must be revised since stenosis depends also on the degree of vessel remodelling. The relative contribution of these factors to stenosis remains unclear and it is likely that the balance between plaque growth and remodelling varies both from patient to patient ${ }^{5}$ and from plaque to plaque. ${ }^{6}$

A terminology has evolved from intravascular studies in which adequate remodelling is applied to the situation where the increase in external area of the vessel neutralises the effect of the increase in plaque size and there is thus no luminal stenosis. Inadequate remodelling is applied to segments where stenosis occurs; in fact this may be because the degree of remodelling is minimal or the plaque burden so great that even a considerable degree of remodelling is negated. Clinical studies in femoral arteries ${ }^{7}$ and in coronary arteries ${ }^{89}$ have also found negative remodelling (or vessel shrinkage), which is a reduction of the cross sectional area of the artery at the site of a plaque. It has been suggested that this process is particularly important in high grade stenoses. ${ }^{11}$

To date studies describing negative remodelling have been performed in vivo with relatively small numbers of plaques and in highly selected lesions (preinterventional). These studies have also been limited by the practical problems of using intravascular ultrasound (for example, non-uniform transducer rotation, eccentric catheter position, acoustic shadowing, and local vasospasm). Pathology studies of postmortem material do not suffer from these limitations but are rarely carried out because of the need to perfuse the arteries at physiological pressures to obtain a lumen shape and size analogous to that which occurs in vivo. Vessel shrinkage in response to plaque growth and the influence of sex has not been studied in detail in the pathological specimens of human coronary arteries. We therefore set out to determine whether negative vessel remodelling (or vessel shrinkage) is seen at postmortem examination in a large unselected collection of coronary artery segments. We also elected to investigate the relative roles of plaque growth and vessel remodelling in the development of luminal stenosis and to determine whether patient factors 


\begin{tabular}{|c|c|c|}
\hline Patient & Number of lesions & Range of remodelling (\%) \\
\hline 1 & 13 & -38 to -49 \\
\hline 2 & 6 & -33 to -105 \\
\hline 3 & 9 & -31 to -105 \\
\hline 4 & 3 & -30 to -27 \\
\hline 5 & 12 & -25 to -60 \\
\hline 6 & 10 & -23 to -8.5 \\
\hline 7 & 10 & -23 to -43 \\
\hline 8 & 11 & -21 to -42 \\
\hline 9 & 7 & -21 to -91 \\
\hline 10 & 14 & -11 to -23 \\
\hline 11 & 8 & -20 to -59 \\
\hline 12 & 8 & -19 to -96 \\
\hline 13 & 10 & -18 to -56 \\
\hline 14 & 8 & -17 to -51 \\
\hline 15 & 7 & -17 to -109 \\
\hline 16 & 7 & -17 to -134 \\
\hline 17 & 7 & -16 to -41 \\
\hline 18 & 5 & -14 to -51 \\
\hline 19 & 9 & -14 to -52 \\
\hline 20 & 3 & -14 to -69 \\
\hline 21 & 8 & -14 to -85 \\
\hline 22 & 6 & -13 to -27 \\
\hline 23 & 5 & -13 to -42 \\
\hline 24 & 7 & -13 to -42 \\
\hline 25 & 10 & -11 to -38 \\
\hline 26 & 8 & -10 to -40 \\
\hline 27 & 14 & -9 to -44 \\
\hline 28 & 6 & -8 to -61 \\
\hline 29 & 10 & -8 to -84 \\
\hline 30 & 10 & -7 to -60 \\
\hline 31 & 17 & -7 to -66 \\
\hline 32 & 8 & -5 to -44 \\
\hline 33 & 10 & -4 to -18 \\
\hline 34 & 9 & -4 to -59 \\
\hline 35 & 7 & -3 to -79 \\
\hline 36 & 6 & -3 to -16 \\
\hline 37 & 5 & 0 to -21 \\
\hline 38 & 4 & 0 to -47 \\
\hline 39 & 13 & 0 to -48 \\
\hline 40 & 8 & 0 to -50 \\
\hline 41 & 5 & 0 to -76 \\
\hline 42 & 3 & 3 to -35 \\
\hline 43 & 13 & 5 to -54 \\
\hline 44 & 5 & 6 to -92 \\
\hline 45 & 6 & 7 to -50 \\
\hline 46 & 7 & 7 to -76 \\
\hline 47 & 8 & 9 to -89 \\
\hline 48 & 4 & 17 to -23 \\
\hline
\end{tabular}

such as age, smoking status, and sex play a role in these processes.

\section{Methods}

HISTOPATHOLOGY

Hearts were obtained after death from 48 patients (24 men and 24 women) who died suddenly and in whom no other cause of death other than coronary artery disease was detectable by detailed necropsy including toxicology.

All hearts were perfusion fixed at physiological pressure with $10 \%$ buffered formalin. After the three major epicardial vessels were dissected out, the tissue was decalcified in formic acid on tissue blocks taken in the transverse plane every $3 \mathrm{~mm}$ through the artery. Tissue was prepared for histological analysis in a standard manner and stained with elastic van Gieson stain.

Because of the problems of measuring plaque size at sites of acute thrombosis, all sites of plaque rupture were excluded and examination was confined to discrete fibrolipid plaques. Also excluded were plaques in which no adjacent disease free segment was available for use as a reference site.

All measurements were taken by computerised planimetry at the plaque cross section with the largest plaque area. For each plaque the following measurements were taken: the area

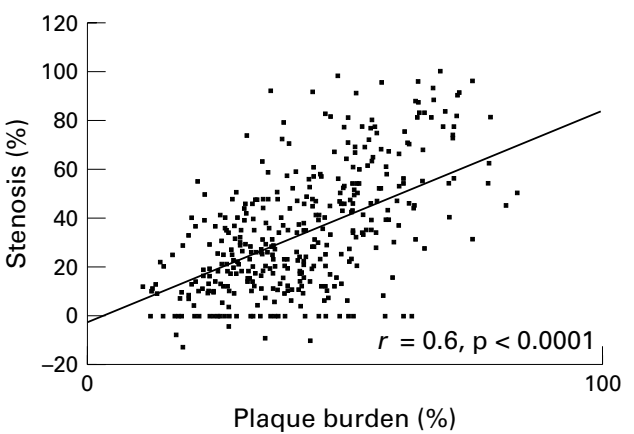

Figure 1 Luminal stenosis against plaque burden.

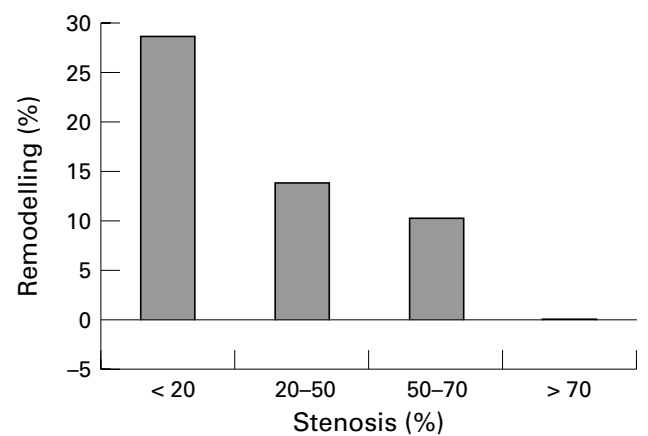

Figure 2 Luminal stenosis against vessel remodelling.

circumscribed by the external elastic lamina (the total vessel cross sectional area), the area bordered by the intima (lumen area), and the area occupied by plaque. Reference cross sectional area and lumen areas were measured at the nearest proximal and distal segments judged to be free of plaque and a mean value was calculated. For each plaque segment the arc of the vessel circumference that was free of disease was measured in degrees.

The percentage luminal stenosis was calculated as ( $1-[$ lumen area/mean of the reference lumen area]) $\times 100 \%$. Positive values thus reflect luminal narrowing and negative values reflect luminal dilatation (for example, in coronary ectasia or aneurysm formation). Percentage vessel remodelling was calculated as ([cross sectional area - mean of the reference cross sectional area]/mean of the reference cross sectional area) $\times 100 \%$. Lesions in which vessel remodelling was $\geqslant 0 \%$ were considered to have positive remodelling and lesions in which remodelling was $<0 \%$ were considered to have negative remodelling. As plaque size varies with vessel size and thus larger vessels tend to have larger plaques, we corrected for vessel size and expressed this as plaque burden, where percentage plaque burden was calculated as (plaque area/cross sectional area) $\times$ $100 \%$. The arc of normal vessel for each site was calculated as (plaque free arc/total vessel circumference) $\times 360^{\circ}$. Lesions in which the arc of normal (plaque free) vessel was $<90^{\circ}$ were considered to have circumferential plaque involvement and those with an arc of normal vessel $\geqslant 90^{\circ}$ were defined as eccentric plaques.

CLINICAL FEATURES

For each patient, age, sex, smoking status (current smoker, former smoker or non-smoker), 
Table 2 Correlation coefficients for multilinear regression modelling of percentage plaque burden and percentage vessel remodelling against percentage luminal stenosis

\begin{tabular}{|c|c|c|c|c|c|c|c|c|c|c|}
\hline & \multicolumn{2}{|c|}{ All lesions $(n=389)$} & \multicolumn{2}{|c|}{$\begin{array}{l}\text { Lesions with stenosis } \\
<25 \%(n=168)\end{array}$} & \multicolumn{2}{|c|}{$\begin{array}{l}\text { Lesions with stenosis } \\
\geqslant 25 \%(n=221)\end{array}$} & \multicolumn{2}{|c|}{$\begin{array}{l}\text { Lesions with negative } \\
\text { remodelling }(n=72)\end{array}$} & \multicolumn{2}{|c|}{$\begin{array}{l}\text { Lesions with positive } \\
\text { remodelling }(n=317)\end{array}$} \\
\hline & Estimate & $p$ Value & Estimate & $p$ Value & Estimate & p Value & Estimate & p Value & Estimate & $p$ Value \\
\hline Plaque burden & 1.0 & 0.0001 & 0.5 & 0.0001 & 0.9 & 0.0001 & 1.0 & 0.0001 & 1.0 & 0.0001 \\
\hline Remodelling & -0.4 & 0.0001 & -0.2 & 0.0001 & -0.1 & 0.09 & -0.6 & 0.12 & -0.4 & 0.0001 \\
\hline
\end{tabular}

Table 3 Characteristics (clinical and pathological) of lesions with positive versus negative remodelling

\begin{tabular}{llll}
\hline & $\begin{array}{l}\text { Lesions with positive } \\
\text { remodelling }(n=317)\end{array}$ & $\begin{array}{l}\text { Lesions with negative } \\
\text { remodelling }(n=72)\end{array}$ & p Value \\
\hline Age (years) & $60.5(6.9)$ & $61.9(5.8)$ & 0.08 \\
Total heart weight $(\mathrm{g})$ & $437.8(101.5)$ & $453.7(139.7)$ & 0.27 \\
Reference XSA (mm) & $8.4(4.2)$ & $11.1(4.8)$ & 0.004 \\
Reference lumen $(\mathrm{mm})$ & $5.3(2.5)$ & $6.4(2.7)$ & 0.03 \\
XSA (mm) & $11.2(4.9)$ & $9.5(3.6)$ & 0.009 \\
Lumen area $\left(\mathrm{mm}^{2}\right)$ & $3.5(2.3)$ & $2.6(2.0)$ & 0.03 \\
Plaque size $\left(\mathrm{mm}^{2}\right)$ & $4.4(2.6)$ & $4.5(1.9)$ & 0.7 \\
Plaque burden $(\%)$ & $40.5(13.5)$ & $47.5(17)$ & 0.002 \\
Stenosis $(\%)$ & $28.9(23)$ & $53.4(24)$ & 0.0001 \\
Arc of normal $\left({ }^{\circ}\right)$ & $102.5(82.5)$ & $56.9(73.8)$ & 0.0001 \\
\hline
\end{tabular}

Data are mean (SD). XSA, cross sectional area.

number of cigarettes smoked, history of hypertension, history of diabetes mellitus, and total heart weight were recorded.

STATISTICAL ANALYSIS

All data are presented as mean (SD). The paired Student's $t$ test was used to analyse categorical variables and continuous variables were analysed by correlation and regression analysis. Multilinear regression modelling was performed using the SPSS statistical programme version 6.0 (SPSS Inc, Chicago, Illinois, USA) to determine the relation between luminal stenosis, plaque burden, and vessel remodelling. A probability value of $\mathrm{p}<0.05$ was considered significant.

\section{Results}

The mean subject age was 60.5 (7.2) years (range 35-69 years). Twenty seven patients were current or former smokers, and 15 had a previous history of hypertension. Only two patients had diabetes mellitus.

A total of 389 discrete coronary artery lesions were examined. A wide spectrum of atherosclerotic disease was seen, both within and between patients (table 1). Mean luminal stenosis was $33.4(25.1) \%$ (range $-12.8 \%$ to $100 \%)$. In 168 (43\%) lesions luminal stenosis was $\leqslant 25 \%$, including five lesions in which stenosis was $<0 \%$ (luminal dilatation) and 39 lesions in which there was no luminal stenosis.

The mean vessel remodelling was 16.8 (24.6) $\%$ (range $-38.0 \%$ to $134.2 \%$ ). In the 389 lesions examined, remodelling was negative in 72 lesions $(18.5 \%)$.

RELATION BETWEEN STENOSIS, PLAQUE BURDEN, AND VESSEL REMODELLING

A positive relation was seen between percentage luminal stenosis and both plaque area and percentage plaque burden $(r=0.3, \mathrm{p}<0.0001$ and $r=0.6, \mathrm{p}<0.0001$, respectively) (fig 1). An inverse relation was found between percentage stenosis and vessel remodelling $(\mathrm{r}=-0.4, \mathrm{p}<0.0001)$ (fig 2). Thus, the lesion with the most negative remodelling had a high grade stenosis (luminal stenosis of $91 \%$ ) and that with the greatest positive remodelling $(+134 \%)$ had a low grade luminal stenosis $(16.7 \%)$. The vessel remodelling of the lesion with the maximal luminal stenosis $(100 \%)$ was $-25 \%$.

Multilinear regression modelling (table 2) of the total plaque population showed that luminal stenosis was equal to 1.0 (plaque burden) -0.4 (vessel remodelling). Both plaque burden and vessel remodelling coefficients were highly significant $(\mathrm{p}<0.0001)$. It was also seen that in lesions in which the stenosis was greater than $25 \%$ the correlation coefficient for remodelling was notably reduced compared with that for plaque burden and was no longer significant (table 2).

Comparison of percentage vessel remodelling in these two groups (stenosis $\leqslant 25 \%$ or $>25 \%$ ) showed that outward remodelling was significantly greater in lesions in which there were low grade stenoses $(\leqslant 25 \%)$ than in the remaining lesions $(25.9$ (26)\% $v 10.0$ (21.1)\%, respectively, $\mathrm{p}<0.0001)$.

An inverse relation was seen between percentage luminal stenosis and the arc of normal artery $(r=-0.6, \mathrm{p}<0.0001)$, and a positive, but very poor relation seen between percentage remodelling and the arc of normal artery $(r=0.14, \mathrm{p}=0.02)$.

One hundred and eighty four lesions were considered to have circumferential plaque involvement and 205 eccentric plaques. Vessel wall remodelling was significantly reduced in segments with circumferential plaques (remodelling $12.7 \quad(24.5) \% \quad v \quad 20.7 \quad(24.3) \% \quad$ in eccentric plaques, $\mathrm{p}=0.001)$.

\section{CORRELATION WITH CLINICAL FEATURES}

Vessel wall remodelling failed to show any significant relation to patient age, sex, or smoking status.

No significant correlation was seen between patient age and any of the lesion characteristics assessed.

POSITIVE VERSUS NEGATIVE VESSEL REMODELLING The lesion characteristics for positive compared with negative remodelling are shown in table 3. Lesions with negative remodelling had a significantly greater plaque burden and luminal stenosis, and a reduced arc of normal arterial segment. The reference cross sectional area was significantly greater in these lesions. No association was seen between positive or negative remodelling and any of the clinical features assessed, including a history of hypertension. Similar results were achieved when negative remodelling was defined as vessel remodelling $\leqslant 0 \%$ or $\leqslant-5 \%$. 


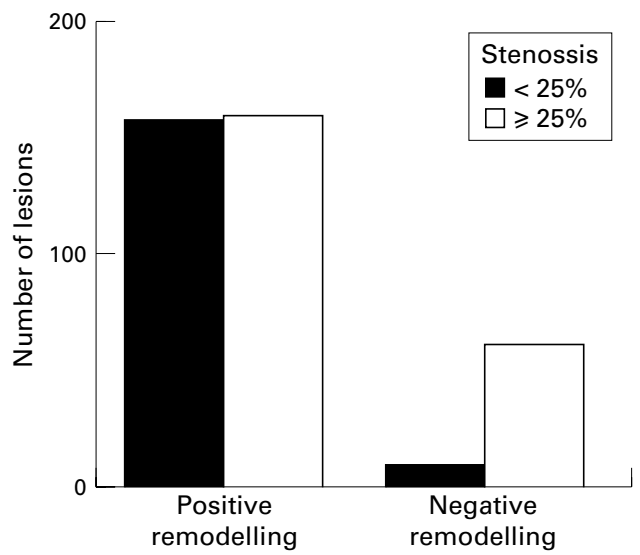

Figure 3 Number of lesions with positive versus negative remodelling according to percentage luminal stenosis.

A significant association was seen between higher grade stenoses $(>25 \%)$ and negative remodelling; of the 72 lesions with negative remodelling, $62(86 \%)$ had a stenosis of $>25 \%$ versus $10(14 \%)$ with a low grade stenosis of $\leqslant 25 \%(\mathrm{p}<0.0001)$. In comparison, among the 317 lesions with positive remodelling, 158 had low grade stenosis and 159 had higher grade stenosis (fig 3 ).

\section{Discussion}

This pathology study on a large number of coronary artery lesions across a wide spectrum of atherosclerotic disease confirmed that coronary artery stenosis is dependent on both plaque burden and vessel remodelling, such that increasing plaque burden increases stenosis while positive remodelling minimises it. Across the total plaque population both processes are of significance, but there is a closer correlation between stenosis and plaque burden. The ability of remodelling to protect the artery against stenosis appears to be particularly apparent in low grade obstruction (lesions in which luminal stenosis is $\leqslant 25 \%$ ). In higher grade stenoses this relation is lost and instead it appears that the development of luminal stenosis is related more to plaque burden. There are two possible explanations for the lack of remodelling in moderate to high grade stenotic lesions. One is that these lesions have never undergone compensatory enlargement to any significant degree and thus stenoses have developed as the plaque has evolved; the second is that remodelling occurs in the early stages of plaque growth, but later as this process is overcome, it is no longer able to prevent the development of luminal stenosis. The bar chart of stenosis against remodelling (fig 2) supports the first hypothesis as some remodelling should be apparent in these moderate to high grade lesions if late failure were the explanation.

In this pathology study of remodelling in human coronary arteries, $18 \%$ of the lesions examined had evidence of negative remodelling (vessel shrinkage). This number is much lower than that reported from most in vivo studies (which are likely to be skewed by the selective nature of the lesions studied) and is probably a more accurate reflection of plaque populations as a whole. Indeed, among lesions with greater luminal stenoses (and thus more equivalent to the intravascular ultrasound target lesion), vessel shrinkage was more frequent (fig 3). In lesions with vessel shrinkage, stenosis was significantly related to plaque burden but not to coronary artery remodelling. In addition, lesions with negative remodelling had a significantly greater reference cross sectional area. The larger reference vessel area and lumen area of lesions with negative remodelling may therefore imply that negatively remodelled lesions are located at more proximal sites in the coronary artery. Indeed, Pasterkamp and colleagues ${ }^{5}$ found evidence to suggest that positive remodelling was enhanced in small vessels, suggesting that this process was greatest in distal rather than proximal sites in the coronary tree. However, the converse cannot be assumed (that is, that vessel shrinkage predominates at proximal sites). An alternative explanation is that these differences in reference vessel area result from pre- or poststenotic dilatation of the reference site. Negative remodelling may therefore not be an active process of vessel shrinkage, but instead a reflection of increases in the reference segments adjacent to high grade stenoses. The vessel shrinkage at target sites in in vivo studies $^{10}{ }^{11}$ may be more apparent than real.

Many theories have been postulated on the mechanisms of compensatory enlargement (for example, endothelium dependent arterial dilatation $^{12}{ }^{13}$ and focal vessel wall thinning secondary to metalloproteinase secretion ${ }^{14}{ }^{15}$ ), but these would take place over some time. Failure of compensatory enlargement may result from sudden plaque growth, which causes stenosis to develop relatively rapidly. In support of this view a high proportion of high grade stenotic lesions have been shown to have an episode of healed plaque disruption. ${ }^{16}$ These episodes would trigger accelerated smooth muscle cell proliferation within the plaque, ${ }^{17}$ causing sudden increases in plaque size, which may be too rapid for compensatory dilatation to overcome.

Lesions in which there was eccentric plaque exhibited much greater vessel wall remodelling than those in which the plaque distribution was circumferential. This has been described before using in vivo techniques. ${ }^{18}$

There was no association between vessel remodelling and any of the other clinical features assessed. In particular there was no influence of sex on remodelling despite an association between male sex and increased plaque area. Our findings are similar to those of in vivo studies that have also failed to find an association between remodelling and sex or other clinical variables. ${ }^{81018}$ Indeed remodelling was seen not only to vary greatly from patient to patient irrespective of age, sex, and smoking status, but also to vary from lesion to lesion within the same artery. It is likely that lesion specific features such as local wall stress, vessel bifurcation, ${ }^{19}$ the rate of plaque growth, and perhaps calcification ${ }^{8}$ are more important in determining the remodelling response to plaque growth than patient specific features such as sex. 
CONCLUSIONS

Positive arterial remodelling is able to negate the stenosing effect of an increase in plaque size, particularly when there is a residual segment of normal arterial wall. Significant coronary artery stenosis arises from a failure of this arterial remodelling in the face of a large plaque burden. This failure is not related to patient specific factors but instead may arise from plaque specific factors such as an episode of sudden plaque growth.

AV is supported by a Clinical Training Fellowship from the Medical Research Council, London, UK.

1 Glagov S, Weisenberg E, Zarins CK, et al. Compensatory enlargement of human atherosclerotic coronary arteries. $N$ Engl f Med 1987;316:1371-5.

2 McPherson DD, Sirna SJ, Hiratzka LF, et al. Coronary arterial remodelling studied by high-frequency epicardial echocardiography: an early compensatory mechanism in patients with obstructive coronary atherosclerosis. $\mathcal{f} \mathrm{Am}$ Coll Cardiol 1991;17:79-86.

3 Hermiller JB, Tenaglia AN, Kisslo KB, et al. In vivo validation of compensatory enlargement of atherosclerotic coronary arteries. Am f Cardiol 1993;71:665-8.

4 Losordo DW, Rosenfield K, Kaufman J, et al. Focal compensatory enlargement of human arteries in response to progressive atherosclerosis. In vivo documentation using intravascular ultrasound. Circulation 1994;89:2570-7.

5 Pasterkamp G, Borst C, Post MJ, et al. Atherosclerotic arterial remodelling in the superficial femoral artery. Individual variation in local compensatory enlargement response. Cirvariation in local compens

6 Varnava A. Coronary artery remodelling. Heart 1998;79: 109-10.

7 Pasterkamp G, Wensing PJ, Post MJ, et al. Paradoxical arterial wall shrinkage may contribute to luminal narrowing of human atherosclerotic femoral arteries. Circulation 1995, 91:1444-9.
8 Mintz GS, Kent KM, Pichard AD, et al. Contribution of inadequate arterial remodelling to the development of focal inadequate arterial remodelling to the development of focal
coronary artery stenoses. An intravascular ultrasound coronary artery stenoses. An in
study Circulation 1997;95:1791-8.

9 Nishioka T, Luo H, Eigler NL, et al. Contribution of inadequate compensatory enlargement to development of human coronary artery stenosis: an in vivo intravascular ultrasound study. F Am Coll Cardiol 1996;27:1571-6.

10 Smits PC, Bos L, Quarles UM, et al. Shrinkage of human coronary arteries is an important determinant of de novo atherosclerotic luminal stenosis: an in vivo intravascular ultrasound study. Heart 1998;79:143-7.

11 Wong C, Porter TR, Xie F, et al. Segmental anlysis of coronary arteries with equivalent plaque burden by intravascular ultrasound in patients with and without angiographically significant coronary artery disease. Am f Cardiol 1995;76: significan

12 Tronc F, Wassef M, Eposito B, et al. Role of NO in flow-induced remodelling of the rabbit common carotid artery. Arterioscler Thromb Vasc Biol 1996;16:1256-62.

13 Lerman A, Cannan CR, Higano SH, et al. Coronary vascular remodelling in association with endothelial dysfunction. Am f Cardiol 1998;81:1105-9.

14 Abbruzzese TA, Guzman RJ, Martin RL, et al. Matrix metalloproteinase inhibition limits arterial enlargement in a rodent arteriovenous fistula model. Surgery 1998;124:32834 .

15 Bassiouny HS, Song RH, Hong XF, et al. Flow regulation of 72-kD collagenase IV (MMP-2) after experimental arterial $72-\mathrm{kD}$ collagenase IV (MMP-2) aft
injury. Circulation $1998 ; 98: 157-63$.

16 Mann J, Davies MJ. Mechanisms of progression in native coronary artery disease: role of healed plaque disruption. Heart 1999;82:265-8.

17 Flugelman MY, Virmani R, Correa E. Smooth muscle cell abundance and fibroblast growth factors in coronary lesions of patients with non fatal unstable angina: a clue to the mechanism of transformation from the stable to the unstable clinical state. Circulation 1993;88:2493-500.

18 Von Birgelen C, Mintz GS, de Vrey EA, et al. Atherosclerotic coronary lesions with inadequate compensatory enlargement have smaller plaque and vessel volumes: enlargement have smaller plaque and vessel volumes: sound in vivo. Heart 1998;79:137-42.

19 deBakey ME, Lawrie GM, Glaeser DH. Patterns of atherosclerosis and their surgical significance. Ann Surg 1985; 201:115-31.

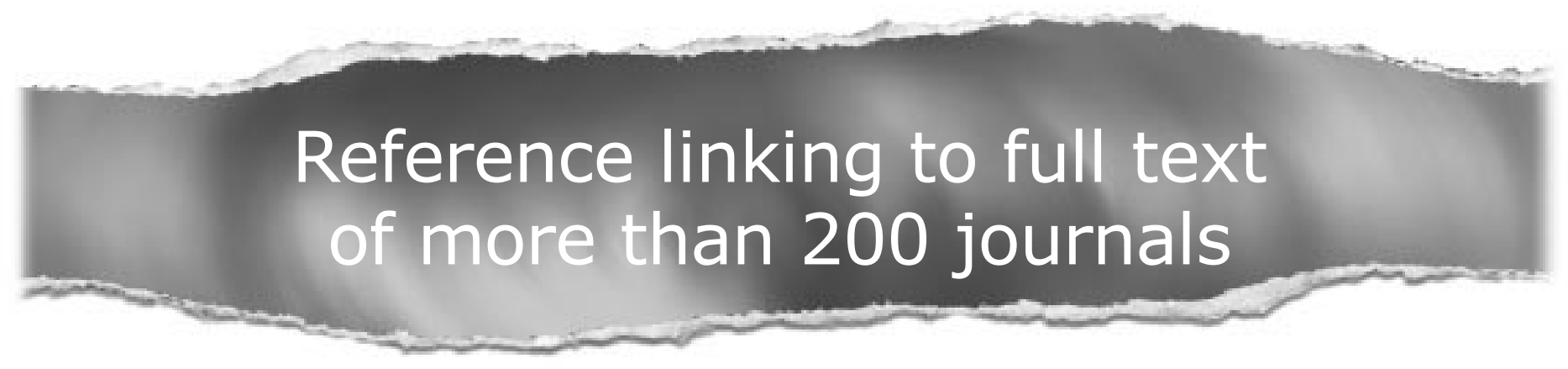

Toll free links

You can access the FULL TEXT of articles cited in Heart online if the citation is to one of the more than 200 journals hosted by HighWire (http://highwire.stanford.edu) without a subscription to that journal. There are also direct links from references to the Medline abstract for other titles.

www.heartjnl.com 